\title{
Tratamento do Dedo em Gatilho
}

\section{Trigger Finger Treatment}

\author{
João Carlos Belloti ${ }^{1 \oplus}$ Edson Sasahara Sato ${ }^{1 \oplus}$ Flavio Faloppa $^{1 \oplus}$
}

${ }^{1}$ Departamento de Ortopedia e Traumatologia, Escola Paulista de Medicina, Universidade Federal de São Paulo, São Paulo, SP, Brasil

Rev Bras Ortop 2022;57(6):911-916.
Endereço para correspondência Edson Sasahara Sato, PhD, Departamento de Ortopedia e Traumatologia, Universidade Federal de São Paulo, Escola Paulista de Medicina, Rua Borges Lagoa, 786, São Paulo, SP, 04038-001, Brasil (e-mail: edsonsasahara@gmail.com).

\section{Resumo \\ Palavras-chave \\ - dedo em gatilho/ diagnóstico \\ - dedo em gatilho/ terapia \\ - dedo em gatilho/ cirurgia \\ - tenossinovite}

O dedo em gatilho é uma afecção frequente. Não obstante a tenossinovite e a alteração da polia A1 serem identificados como fatores desencadeantes, não há consenso sobre a verdadeira causa na literatura, sendo que a sua verdadeira etiologia permanece desconhecida. O diagnóstico é puramente clínico na maior parte das vezes. Ele depende unicamente da existência do travamento do dedo no decorrer da movimentação flexão ativa. O tratamento do dedo em gatilho geralmente se inicia com intervenções não cirúrgicas que são instituídas por pelo menos 3 meses. Nos pacientes em quem haja apresentação inicial com deformidade em flexão ou incapacidade de flexão do dedo, pode haver indicação mais precoce do tratamento cirúrgico em razão da intensidade do quadro álgico e da incapacidade funcional do paciente.

No presente artigo de revisão, apresentaremos as modalidades e o nosso algoritmo para o tratamento do dedo em gatilho.

Trigger finger is a frequent condition. Although tenosynovitis and the alteration of pulley $\mathrm{A} 1$ are identified as triggering factors, there is no consensus on the true cause in the literature, and its true etiology remains unknown. The diagnosis is purely clinical most of the time. It depends solely on the existence of finger locking during active bending movement. Trigger finger treatment usually begins with nonsurgical interventions that are instituted for at least 3 months. In patients with initial presentation with flexion deformity or inability to flex the finger, there may be earlier indication of surgical treatment due to pain intensity and functional disability.

In the present review article, we will present the modalities and our algorithm for the treatment of trigger finger.

\section{Introdução}

Notta $^{1}$ foi o primeiro a descrever o dedo em gatilho como uma patologia desencadeada por alterações no tendão flexor e sua bainha. Hueston et al. ${ }^{2}$ demonstraram em um estudo que $o$ arranjo espiral na arquitetura das fibras intratendíneas leva à formação de uma nodulação ao passar por um ponto de estenose. recebido

10 de Janeiro de 2020

aceito

15 de Abril de 2020

Publicado on-line

Setembro 20, 2022
DOI https://doi.org/

10.1055/s-0040-1713765.

ISSN 0102-3616.
(C) 2022. Sociedade Brasileira de Ortopedia e Traumatologia. All rights reserved.

This is an open access article published by Thieme under the terms of the Creative Commons Attribution-NonDerivative-NonCommercial-License, permitting copying and reproduction so long as the original work is given appropriate credit. Contents may not be used for commercial purposes, or adapted, remixed, transformed or built upon. (https://creativecommons.org/ licenses/by-nc-nd/4.0/)

Thieme Revinter Publicações Ltda., Rua do Matoso 170, Rio de Janeiro, RJ, CEP 20270-135, Brazil 
Brozovich et al. ${ }^{3}$ descreveram alterações histológicas na polia A1, que sofre uma metaplasia com substituição de fibroblastos por condrócitos, sendo um dos motivos de aumento de pressão entre o tendão e o túnel osteofibroso no dedo em gatilho.

Não obstante a tenossinovite e a alteração da polia A1 serem identificados como fatores desencadeantes, não há consenso na literatura sobre a verdadeira causa, sendo que a sua verdadeira etiologia permanece desconhecida. ${ }^{4}$

A definição do dedo em gatilho é que se trata de uma afecção na qual o tendão flexor tem o seu deslizamento bloqueado ao passar no sistema de polias, notadamente na polia $\mathrm{A} 1$, não conseguindo mais excursionar e retornar à posição inicial, impedindo que o dedo faça o seu movimento natural de flexão e extensão.

Essa afecção acomete de 2 a 3\% da população, mais na $6^{a}$ década de vida. ${ }^{5} \mathrm{O}$ dedo mais afetado é o anular, seguido pelos dedos médio, polegar, mínimo e indicador. A mão dominante é acometida em $70 \%$ das vezes. As mulheres têm o dobro de chance de terem dedo em gatilho do que os homens. ${ }^{6}$

Pacientes com diabetes têm 15\% a mais de chance de desenvolver dedo em gatilho do que a população em geral. Entretanto, quando tratados pela cirurgia, têm a mesma evolução que pacientes não diabéticos, assim como não têm maior chance de apresentarem múltiplos dedos com gatilho. ${ }^{7}$

Por vezes, o dedo em gatilho tem sido associado com a cirurgia de liberação do túnel do carpo, corroborando com esta concepção, numa revisão sistemática da literatura, Lin et al., ${ }^{8}$ que descreveram um índice médio de 8,5\% de desenvolvimento da afecção após o procedimento para abertura do retináculo flexor.

O dedo em gatilho também acomete as crianças, sendo comumente denominado como "gatilho congênito", tendo diferenças na apresentação clínica.

O "gatilho congênito" no polegar é 10 vezes mais comum do que o nos outros dedos, e os pacientes geralmente apresentam $\sim 2$ anos de idade. ${ }^{9}$ A incidência de "gatilho congênito" é de 3,3 por 1.000 nascidos vivos. ${ }^{10}$ Apesar da etiologia do "gatilho congênito" ser desconhecida, sabe-se que se trata de uma afecção adquirida. Autores avaliaram 7.000 recém-nascidos e não encontraram nenhum dedo em gatilho. ${ }^{11}$

\section{Quadro clínico}

O sintoma geralmente inicia com dor na área coincidente à polia A1 nos dedos. A ocorrência surge gratuitamente ou posterior a uma ação manual continuada preambular. A dor pode continuar por meses sem alteração na mobilidade, ou progredir para um bloqueio do movimento do dedo, inicialmente pela manhã, persistindo nesta situação ou avançando para um gatilho mais constante, a toda flexão ativa do dedo. Similarmente, o fenômeno do gatilho pode ter início repentino, sem um pródromo doloroso. Esta manifestação sobrechega meramente em um dígito ou em mais de um ao mesmo tempo.

Quinnell ${ }^{4}$ estudou os resultados da injeção de hidrocortisona na polia $\mathrm{A} 1 \mathrm{em} 48$ dedos em gatilho de 43 pacientes. Ele dividiu os diferentes grupos de dedos em gatilho em cinco tipos, relacionando-os com a flexão e extensão, sendo o tipo "0" com movimento normal, tipo "I" o gatilho esporádico,
Tabela 1 Classificação para dedo em gatilho segundo Green DP (Green DP, personal communication, 1997) ${ }^{12}$

\begin{tabular}{|l|l|}
\hline Tipo & Característica clínica \\
\hline I (pré-gatilho) & $\begin{array}{l}\text { Dor; história de travamento, mas não } \\
\text { demonstrável no exame clínico; sensi- } \\
\text { bilidade a palpação acima da polia A1 }\end{array}$ \\
\hline II (ativo) & $\begin{array}{l}\text { Travamento demonstrável, mas o } \\
\text { paciente consegue estender ativamente } \\
\text { o dedo }\end{array}$ \\
\hline III (passivo) & $\begin{array}{l}\text { Travamento demonstrável necessitando } \\
\text { de extensão passiva (grau IIIA) ou } \\
\text { impossibilidade de flexão ativa do dedo } \\
\text { (grau IIIB) }\end{array}$ \\
\hline IV (contratura) & $\begin{array}{l}\text { Travamento demonstrável com uma } \\
\text { contratura em flexão da articulação } \\
\text { interfalângica proximal }\end{array}$ \\
\hline
\end{tabular}

tipo "II" o gatilho corrigível ativamente, tipo "III" o gatilho corrigivel apenas passivamente, e o tipo "IV" com deformidade fixa. Esta classificação foi modificada por Green $\mathrm{DP}^{12}$ conforme apresentado na - Tabela 1.

É interessante destacar que o dedo em gatilho se trata de um fenômeno que sobrevém unicamente no decurso da flexão ativa do dedo.

O destravamento do dedo pode sobrevir do movimento ativo ou necessitar de uma força externa para tal fim.

Fortuitamente, o dedo apresentar-se-á flexionado permanentemente com impedimento tanto na extensão ativa quanto passiva, tendo o desbloqueio inexecutável, não obstante a energia praticada. Outras vezes estará estendido perenemente, tendo impedimento na flexão ativa.

Ocasionalmente, a articulação interfalângica proximal progride para uma contratura em flexão, habitualmente corrigivel pela extensão passiva, não obstante a dor relacionada à retificação do dedo.

Ademais, o dedo em gatilho pode estar correlacionado a doenças de depósito (sarcoidose, amiloidose), moléstias com alteração do metabolismo (hipotireoidismo, diabetes), assim como a enfermidades autoimunes (artrite reumatoide, lúpus). ${ }^{13}$

\section{Diagnóstico}

O diagnóstico é clínico na maior parte das vezes. Ele depende unicamente da existência do travamento do dedo no decorrer da movimentação ativa.

Na hipótese de o dedo apresentar-se estendido ou flexionado permanentemente, sendo impossibilitado de completar a flexão ativa ou a extensão passiva, respectivamente nesta ordem, a determinação do diagnóstico será mais intrincada.

No dígito estendido com impraticabilidade na flexão ativa, é imperioso descartar uma lesão fechada dos tendões flexores. Nesta situação, após estabilização da falange média, o paciente geralmente será capaz de flexionar ativamente a falange distal, comprovando sua integridade. Outra possibilidade a ser diferenciada será a de contratura em extensão por dano articular, sendo a radiografia interessante nesta situação. 
Já em um dedo flexionado persistentemente, com incapacidade na extensão passiva, será imprescindível diferenciar de uma aderência tendinosa dos tendões flexores ou da contratura em flexão do dedo.

Na incerteza, o exame de ultrassom mostrará o tendão flexor e suas alterações morfológicas, assim como o espessamento da polia flexora. ${ }^{14}$

\section{Tratamento}

O tratamento do dedo em gatilho geralmente se inicia com intervenções não cirúrgicas que são instituídas por pelo menos 3 meses. Nos pacientes em quem haja apresentação inicial com deformidade em flexão ou incapacidade de flexão do dedo, pode haver indicação mais precoce do tratamento cirúrgico em razão da intensidade do quadro álgico e da incapacidade funcional do paciente.

\section{Métodos não cirúrgicos}

\section{Fisioterapia}

Salim et al., ${ }^{15}$ em um estudo comparando a eficácia da infiltração com corticosteroide versus fisioterapia, a fisioterapia atingiu $68,6 \%$ de sucesso versus $97 \%$ da infiltração no tratamento do dedo em gatilho.

Watanabe et al., ${ }^{16} \mathrm{em}$ um estudo, realizando fisioterapia feita pelas próprias mães dos pacientes com "polegar em gatilho congênito", tiveram como resultado a taxa de cura de $80 \%$ para os estágios 2 (gatilho que destrava no movimento ativo) e de $25 \%$ para o estágio 3 (gatilho que destrava somente no movimento passivo).

\section{Imobilização}

A imobilização foi descrita por Patel et al. ${ }^{17}$ por meio de órtese que mantinha a articulação metacarpofalângica em $10^{\circ}$ a $15^{\circ}$ de flexão com as articulações interfalângicas livres por um tempo médio de 6 semanas. 0 tratamento nos dedos indicador, médio anular e mínimo foi bem sucedido em $66 \%$, e nos polegares em $50 \%$.

\section{Infiltração}

A infiltração com corticosteroide é a modalidade de tratamento não cirúrgico mais utilizada, sendo descrita por inúmeros autores. ${ }^{18-22}$.

Sato et al., ${ }^{18}$ em um estudo prospectivo randomizado, comparando os resultados do tratamento do dedo em gatilho pela infiltração versus liberação percutânea versus cirurgia aberta, obtiveram $86 \%$ de cura com a injeção de metilpredinisolona.

Mardani-Kivi et al. ${ }^{19}$ compararam os resultados da infiltração com e sem o auxílio de ultrassom, tendo $94 \%$ de sucesso igualmente em ambos os grupos.

Apesar dos resultados animadores com glucocorticóides, é necessário cautela nos pacientes diabéticos, que podem ter importante elevações nos níveis glicêmicos. ${ }^{20}$

Roberts et al. ${ }^{21}$ compararam os resultados da infiltração com triancinolona, dexametasona e metilpredinisolona, e concluíram que os dedos em gatilho tratados com triancino-

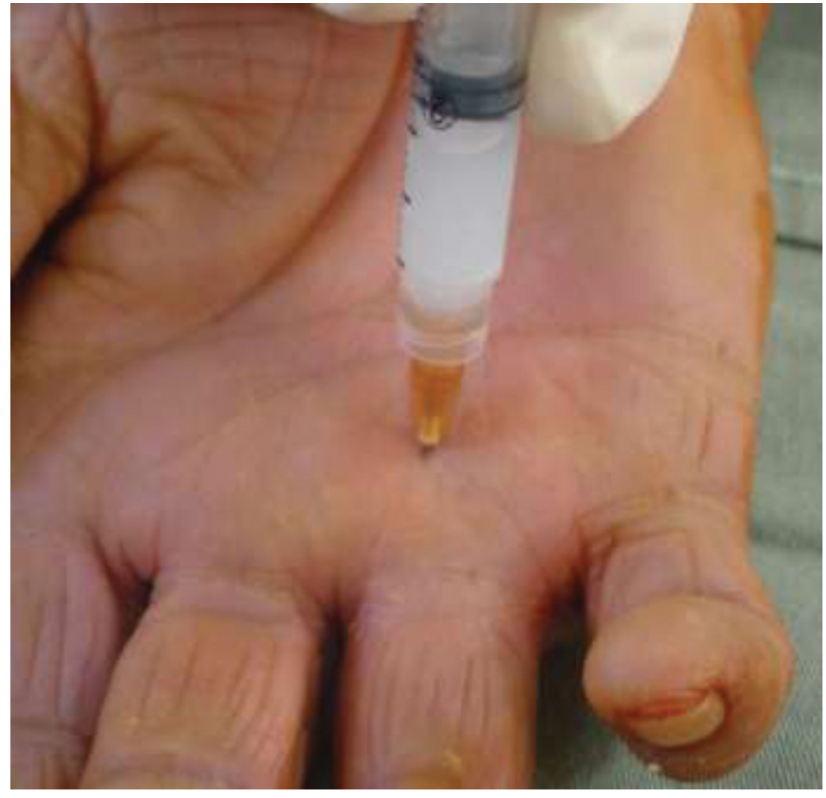

Fig. 1 Injeção de corticosteroide no interior do túnel osteofibroso da polia A1.

lona têm maior chance de necessitar de uma segunda injeção do que os tratados com metilpredinisolona. ou dexametasona.

Newport et al., ${ }^{22}$ em estudo retrospectivo, analisaram a segurança e a eficácia do tratamento do dedo em gatilho, inicialmente por meio da infiltração de betametasona. Os autores examinaram 235 pacientes com 338 dedos em gatilho, $71 \%$ na mão direita, sendo $63 \%$ mulheres e $37 \%$ homens. 0 período de seguimento médio foi de 35 meses. Quanto aos resultados, $49 \%$ melhoraram após uma infiltração, $23 \%$ após 2 infiltrações e $5 \%$ após 3 infiltrações. 0 restante dos pacientes que não melhoraram, totalizando $33 \%$ deles, foram submetidos ao tratamento operatório aberto, através da liberação cirúrgica da polia A1. Segundo estes autores, o tratamento inicial do dedo em gatilho deveria ser com a infiltração de corticosteroide e a sua utilização prévia não prejudicaria o resultado de um tratamento operatório caso este fosse necessário.

Nossa preferência é por até duas infiltrações com intervalo de no mínimo 1 mês, utilizando acetato de metilprednisolona ou dipropionato de betametasona no interior do túnel osteofibroso.

\section{Técnica}

Nós realizamos uma anestesia local com $2 \mathrm{ml}$ de lidocaína a $0,5 \%$, subcutânea, no local coincidente com a polia A1 do dedo afetado. Aproximadamente após 5 minutos, introduzimos a agulha no tendão flexor, através da polia A1. Recuamos a agulha até sentir diminuição da resistência no êmbolo da seringa para então injetar o corticoide. Preferimos utilizar acetato de metilprednisolona ou dipropionato de betametasona (-Figura 1).

\section{Métodos Cirúrgicos}

A terapêutica cirúrgica usualmente é indicada quando houver falha do tratamento não cirúrgico. 
A cirurgia consiste na incisão da polia A1. Ela pode ser executada na forma tradicional aberta ou por via percutânea.

\section{Método percutâneo}

Eastwood et al. ${ }^{23}$ foram os pioneiros na liberação percutânea, apresentando $94 \%$ de resultados satisfatórios.

Sato et al. ${ }^{24}$ realizaram a liberação percutânea do dedo em gatilho, tendo $96 \%$ de remissão, com recidiva nos pacientes do tipo I de Quinnell. Eles concluíram que o gatilho esporádico (tipo I), que não ocorre a toda flexão do dedo, não seria indicado para a liberação percutânea, uma vez que a extinção do travamento é o que indica o sucesso da cirurgia.

Já em um segundo estudo, Sato et al. ${ }^{18}$ excluíram o gatilho do tipo I, tendo $100 \%$ de cura com a liberação percutânea.

Bain et al. ${ }^{25}$ verificaram que o feixe neurovascular do polegar situa-se a $2 \mathrm{~mm}$ da polia A1, ficando mais vulnerável a lesão pela agulha. Apesar disso, Sato et al. ${ }^{18}$ realizaram a liberação percutânea no polegar e não tiveram lesão de nervos digitais.

Por se tratar de um método fechado, autores como Lee et al. ${ }^{26}$ compararam a liberação percutânea com e sem auxílio de ultrassom e concluíram que a visualização da polia $\mathrm{A} 1$ pelo exame de imagem diminui a possibilidade de incisões incompletas.

\section{Técnica}

O paciente é posicionado mantendo a mão afetada em supinação. Com uma caneta marcadora de pele, fazemos um traço sobre o eixo longitudinal do dedo. Infiltram-se $2 \mathrm{ml}$ de anestésico local (lidocaína a $2 \%$, sem vasoconstritor) no tecido subcutâneo e ao redor da polia A1 ( - Figura 2a). As articulações interfalangianas e as metacarpofalangianas dos dedos deverão ficar em extensão total com o objetivo de deslocar dorsalmente os feixes neurovasculares, reduzindo a possibilidade de lesionar essas estruturas durante o procedimento. 0 bisel da agulha deve ser posicionado no sentido do seu corte paralelamente ao eixo longitudinal do dedo (-Figura $2 \mathbf{b}$ ). Introduz-se uma agulha hipodérmica $40 \times 12$ perpendicularmente à pele, no local correspondente à polia A1. Confirma-se o posicionamento da agulha no tendão flexionando o dedo e observando o deslocamento concomitante da agulha ( - Figura 2c). Em seguida, é feita a retração da agulha até que ocorra a parada da oscilação concomitante desta com a movimentação passiva do dedo. Realizam-se movimentos longitudinais no sentido da polia, a fim de seccioná-la ( - Figura 2d). Solicita-se ao paciente que realize movimentos ativos de flexoextensão do dedo operado para confirmar se houve liberação total da polia. Caso necessário, repetem-se os movimentos longitudinais com a agulha, até que se verifique a liberação completa do gatilho. A seguir, retira-se a agulha, finalizando o procedimento, e realiza-se o curativo, sem necessidade de imobilização. Os pacientes são orientados a evitar atividades manuais e a realizar crioterapia com uma bolsa contendo pedras de gelo no dia da realização do procedimento.

\section{Método aberto}

A cirurgia aberta é o método cirúrgico mais clássico e usualmente evidenciado como sendo um método com altos índices de resultados satisfatórios.

Turowski et al. ${ }^{27}$ mostraram resultados satisfatórios com a cirurgia aberta de $97 \%$.

Leung et al. ${ }^{28}$ realizaram a liberação aberta de 161 polegares com "gatilho congênito", obtendo 95\% de resultados satisfatórios.

\section{Técnica}

Paciente em decúbito dorsal, após anestesia. Realiza-se uma incisão cutânea transversal, no local correspondente à polia flexora. Os nervos digitais de cada lado são identificados e afastados delicadamente e a polia flexora é visualizada. (-Figura 3a) A polia é incisada no seu sentido longitudinal
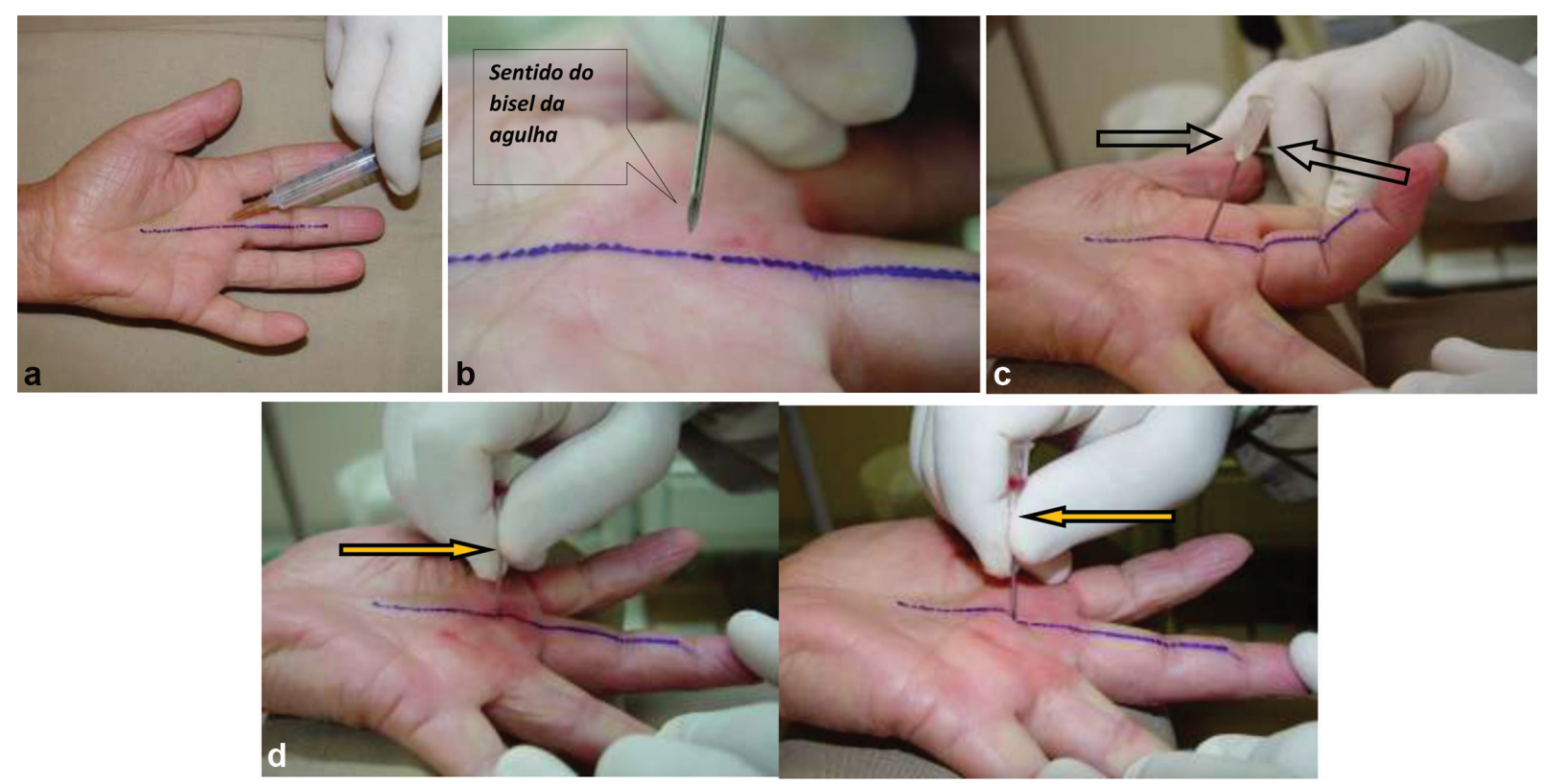

Fig. 2 (a) Anestesia da pele e do tecido celular subcutâneo, com o eixo longitudinal do dedo demarcado. (b) Posicionamento correto do bisel da agulha no momento de sua introdução. (c) Deslocamento da agulha ao se flexionar passivamente o dedo. (d) Seccionando-se a polia A1. 

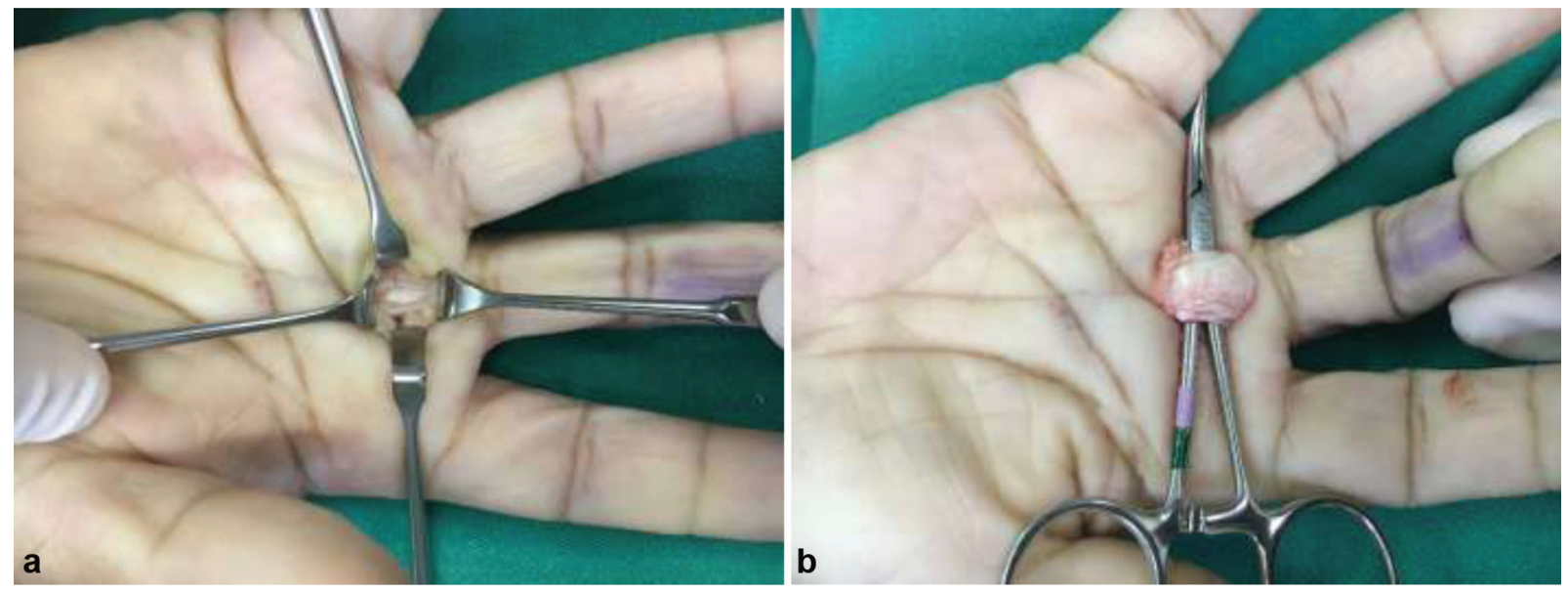

Fig. 3 (a) Incisão e abertura da polia flexora. (b) Tendão exposto.

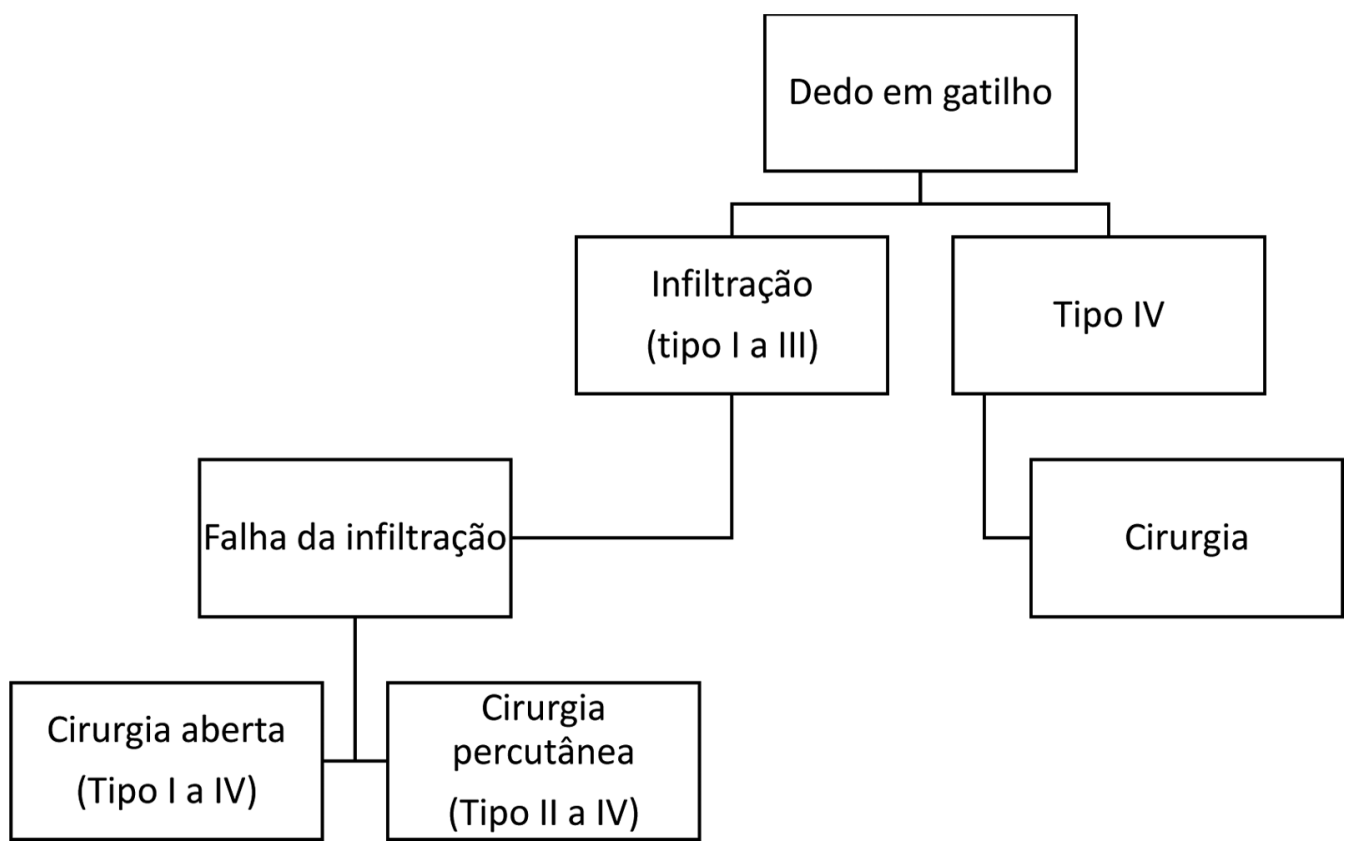

Fig. 4 Algoritmo do tratamento do dedo em gatilho.

e o tendão é exposto (-Figura 3b-c). Após a sutura da pele, cobre-se a ferida com curativo estéril, sem imobilização, por $\sim 1$ uma semana.

\section{Considerações Finais}

Amirfeiz et al. ${ }^{29}$ realizaram uma revisão sistemática comparando os tratamentos para o dedo em gatilho em adultos, e concluíram que a infiltração com corticosteroide como primeiro tratamento é uma opção razoável. A liberação percutânea realizada por pessoa treinada é segura. Na falha da infiltração, os métodos cirúrgicos são indicados.

Nós concordamos que a infiltração deve ser indicada como primeira opção terapêutica; contudo, nos pacientes diabéticos, que podem ter sua glicemia elevada consideravelmente, temos que ter cautela, principalmente nos doentes com níveis glicêmicos não contrabalançados pela medicação.

Fiorini et al. $^{30}$ realizaram uma revisão sistemática do tratamento do dedo em gatilho e concluíram que a cirurgia aberta apresenta menor taxa de recidiva quando comparada com os pacientes tratados com infiltração.

Brozovich et al. $^{3}$ assinalaram que de um ponto de vista puramente financeiro, mulheres sem diabetes com polegar em gatilho devem receber até duas infiltrações antes de serem submetidas a liberação percutânea.

Nós acreditamos que apesar dos métodos cirúrgicos (aberto e percutâneo) terem maiores índices de cura quando comparados com a infiltração, conforme nosso estudo ${ }^{18}$ evidenciou, são opções mais caras, e devem ser reservadas para a falha do tratamento não cirúrgico. 
Apresentaremos a seguir nosso algoritmo de tratamento (-Figura 4).

\section{Conflito de Interesses}

Os autores declaram não haver conflito de interesses.

\section{Referências}

1 Notta A. Research on a particular condition of tendon sheaths of the hand, characterized by the development of a nodule in the path of flexor tendons and blocking their movements. Arch Gen Med 1850;4(24):142-161

2 Hueston JT, Wilson WF. The aetiology of trigger finger explained on the basis of intratendinous architecture. Hand 1972;4(03): 257-260

3 Brozovich N, Agrawal D, Reddy G. A Critical Appraisal of Adult Trigger Finger: Pathophysiology, Treatment, and Future Outlook. Plast Reconstr Surg Glob Open 2019;7(08):e2360

4 Quinnell RC. Conservative management of trigger finger. Practitioner 1980;224(1340):187-190

5 Lunsford D, Valdes K, Hengy S. Conservative management of trigger finger: A systematic review. J Hand Ther 2019;32(02): 212-221

6 N JHS, L AHAF, R GVG, da Silveira DCEC, B PN, Almeida SF. Epidemiology of Trigger Finger: Metabolic Syndrome as a New Perspective of Associated Disease. Hand (N Y) 2019; 28:1558944719867135

7 Ho SWL, Chia CY, Rajaratnam V. Characteristics and Clinical Outcomes of Open Surgery for Trigger Digits in Diabetes. J Hand Microsurg 2019;11(02):80-83

8 Lin FY, Wu Cl, Cheng HT. Coincidence or complication? A systematic review of trigger digit after carpal tunnel release. J Plast Surg Hand Surg 2018;52(02):67-73

9 Baek GH, Kim JH, Chung MS, Kang SB, Lee YH, Gong HS. The natural history of pediatric trigger thumb. J Bone Joint Surg Am 2008;90 (05):980-985

10 Kikuchi N, Ogino T. Incidence and development of trigger thumb in children. J Hand Surg Am 2006;31(04):541-543

11 Moon WN, Suh SW, Kim IC. Trigger digits in children. J Hand Surg [Br] 2001;26(01):11-12

12 Wolfe S, Hotchkiss RN, Pederson W, Kozin SH, Cohen M, Eds. Green's operative hand surgery. 7th. ed. Philadelphia: Elsevier; 2017

13 Akhtar S, Bradley MJ, Quinton DN, Burke FD. Management and referral for trigger finger/thumb. BMJ 2005;331(7507): 30-33

14 Mifune Y, Inui A, Sakata R, et al. High-resolution ultrasound in the diagnosis of trigger finger and evaluation of response to steroid injection. Skeletal Radiol 2016;45(12):1661-1667
15 Salim N, Abdullah S, Sapuan J, Haflah NH. Outcome of corticosteroid injection versus physiotherapy in the treatment of mild trigger fingers. J Hand Surg Eur Vol 2012;37(01):27-34

16 Watanabe H, Hamada Y, Toshima T, Nagasawa K. Conservative treatment for trigger thumb in children. Arch Orthop Trauma Surg 2001;121(07):388-390

17 Patel MR, Bassini L. Trigger fingers and thumb: when to splint, inject, or operate. J Hand Surg Am 1992;17(01):110-113

18 Sato ES, Gomes Dos Santos JB, Belloti JC, Albertoni WM, Faloppa F. Treatment of trigger finger: randomized clinical trial comparing the methods of corticosteroid injection, percutaneous release and open surgery. Rheumatology (Oxford) 2012;51(01):93-99

19 Mardani-Kivi M, Karimi-Mobarakeh M, Babaei Jandaghi A, Keyhani S, Saheb-Ekhtiari K, Hashemi-Motlagh K. Intra-sheath versus extra-sheath ultrasound guided corticosteroid injection for trigger finger: a triple blinded randomized clinical trial. Phys Sportsmed 2018;46(01):93-97

20 Elena C, Chiara M, Angelica B, et al. Hyperglycemia and Diabetes Induced by Glucocorticoids in Nondiabetic and Diabetic Patients: Revision of Literature and Personal Considerations. Curr Pharm Biotechnol 2018;19(15):1210-1220

21 Roberts JM, Behar BJ, Siddique LM, Brgoch MS, Taylor KF. Choice of Corticosteroid Solution and Outcome After Injection for Trigger Finger. Hand (N Y) 2019;•••:1558944719855686

22 Newport ML, Lane LB, Stuchin SA. Treatment of trigger finger by steroid injection. J Hand Surg Am 1990;15(05):748-750

23 Eastwood DM, Gupta KJ, Johnson DP. Percutaneous release of the trigger finger: an office procedure. J Hand Surg Am 1992;17(01): 114-117

24 Sato ES, Albertoni WM, Leite VM, Santos JB, Faloppa F. Trigger finger: a prospective analysis of 76 fingers treated surgically by percutaneous release. Rev Bras Ortop 2004;39(06):309-322

25 Bain GI, Turnbull J, Charles MN, Roth JH, Richards RS. Percutaneous A1 pulley release: a cadaveric study. J Hand Surg Am 1995; 20(05):781-784, discussion 785-786

26 Lee SH, Choi YC, Kang HJ. Comparative study of ultrasonographyguided percutaneous A1 pulley release versus blinded percutaneous A1 pulley release. J Orthop Surg (Hong Kong) 2018;26(02): 2309499018772368

27 Turowski GA, Zdankiewicz PD, Thomson JG. The results of surgical treatment of trigger finger. J Hand Surg Am 1997;22(01):145-149

28 Leung OY, Ip FK, Wong TC, Wan SH. Trigger thumbs in children: results of surgical release. Hong Kong Med J 2011;17(05): 372-375

29 Amirfeyz R, McNinch R, Watts A, et al. Evidence-based management of adult trigger digits. J Hand Surg Eur Vol 2017;42(05): 473-480

30 Fiorini HJ, Tamaoki MJ, Lenza M, Gomes Dos Santos JB, Faloppa F, Belloti JC. Surgery for trigger finger. Cochrane Database Syst Rev 2018;2:CD009860 